\title{
INFLUENCE OF THERMODYNAMIC AND STRUCTURAL PARAMETERS OF MULTILAYER FOILS ON SHS PROCESS CHARACTERISTICS
}

\author{
M.V. KRAVCHUK and A.I. USTINOV \\ E.O. Paton Electric Welding Institute, NASU \\ 11 Bozhenko Str., 03680, Kiev, Ukraine. E-mail: office@paton.kiev.ua
}

\begin{abstract}
Characteristics of the process of self-propagating high-temperature synthesis (SHS) to foil with multilayer structure based on elements, capable of formation of intermetallic compounds, are determined by many thermodynamic and structural parameters. This significantly complicates the possibility of prediction of the features of SHS reaction running in them at the change of the foil structure and chemical composition. In the study, analysis of effectiveness of these parameters influence on SHS reaction front temperature and velocity in multilayer foil was performed within the framework of a phenomenological model. It is shown that the velocity of SHS front propagation and heat generation intensity nonmonotonically depend on structural parameters. In the case of $\mathrm{Ni} / \mathrm{Al}$ multilayer foil, structural parameters were established, for which maximum values of heat generation intensity are reached in the foil in SHS process, and the method for their determination was proposed. 8 Ref., 1 Table, 11 Figures.
\end{abstract}

$\boldsymbol{K} \boldsymbol{e} \boldsymbol{y} \boldsymbol{w} \boldsymbol{o r d} \boldsymbol{s}:$ phase transformations, self-propagating high-temperature synthesis, reaction diffusion, multilayer foils, heat conductivity, structural parameters, heat generation intensity

It is known [1-3] that under certain conditions the process of self-propagating high-temperature synthesis (SHS) with intensive heat evolution can be initiated in multilayer foils (MF) based on intermetallic-forming elements. Such MF are regarded [3] as heat sources, which can provide local heating of the joint zone, necessary for realization of the process of material joining by welding or brazing. It is shown that the velocity and temperature of SHS reaction front in MF depend on its chemical composition and structure. In practical terms, it is necessary to know

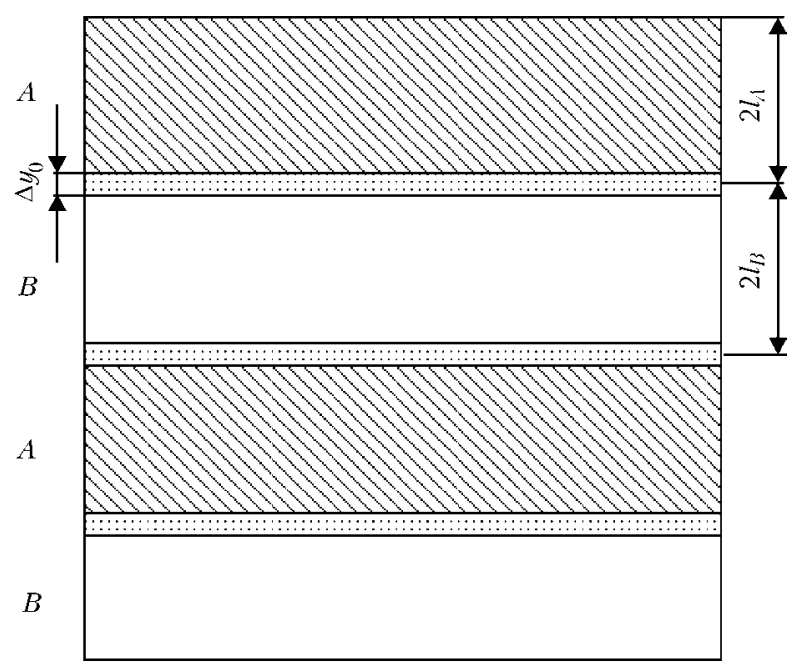

Figure 1. Schematic structure of multilayer foil ๑ M.V. KRAVCHUK and A.I. USTINOV, 2015
MF parameters, which can ensure the maximum level of heat generation in SHS process.

As searching for optimum structure of foil with high reactivity is related to solving a multiparametral problem, it was necessary to assess the effectiveness of the influence of various MF parameters on its characteristics, including not only its microstructural characteristics, but also thermodynamic parameters such as the coefficient of element interdiffusion, diffusion activation energy and thermodynamic stimulus for intermetallic formation.

In this work, the influence of MF structural and thermodynamic characteristics on their reactivity in the mode of stationary SHS process was studied within the framework of a phenomenogical model [4-6].

Investigation procedure. It is known [4] that in the mode of stationary process of SHS front propagation in MF it is possible to correlate MF structural and thermodynamic parameters by simultaneously solving the equations of heat conductivity and diffusion between the reaction elements.

Schematically, MF structure (Figure 1) can be presented as alternation of layers of elements $A$ and $B$, with multilayer period $4 l(\lambda)$, which are separated by an interlayer of thickness $\Delta y_{0}$ of intermetallic phase forming in the foil during its production. In [4] it was shown that the velocity of SHS front propagation in the case of foil equiatomic composition can be presented as 


$$
V=\sqrt{32.32 \frac{a^{2} D_{0} \Delta g}{\left(4 l^{2}-\Delta y_{0}^{2}\right)} \frac{T_{0}\left(k T_{f}+Q\right)}{Q^{2}\left(T_{f}-T_{0}\right)} \exp \left(-\frac{Q}{k T_{f}}\right)},
$$

where $D_{0}$ is the coefficient of interdiffusion; $T_{0}$ is the initial foil temperature; $Q$ is the energy of interdiffusion activation; $\Delta g$ is the thermodynamic stimulus of intermetallic phase formation; $a^{2}$ is the thermal diffusivity; $k$ is the Boltzmann constant; $T_{f}$ is the front temperature which is calculated by the following formula:

$$
T_{f}=T_{0}+\frac{\Delta g}{3 k} f,
$$

where $f=\left(2 l-\Delta y_{0}\right) / 2 l$ is the foil effectiveness (volume fraction of unreacted elements). Thermodynamic stimulus $\Delta g$ is the free energy of intermetallic phase formation from elements $A$ and $B$.

The above equations (1) and (2) show that thermodynamic parameters are determined by elements, which are the basis for multilayer structure formation, and structural parameters are determined by foil preparation conditions. If all the parameters included into equations (1) and (2) have been determined, it is possible to calculate foil reactivity characteristics in the mode of SHS stationary propagation.

For real systems part of these parameters are not determined, and some of them depend on foil preparation conditions and they can change within certain limits. While, for instance, MF multilayer period is determined with sufficient accuracy by studying its cross-sectional microstructure, intermediate layer thickness determination by direct microstructural studies is difficult, as by theoretical estimates it can be equal to several nanometers. However, if two foils are manufactured on the basis of one $A / B$ system under similar conditions with different multilayer periods $\lambda_{1}$ and $\lambda_{2}$ (provided $\lambda_{1}>\lambda_{2}$ ), then, having determined for such foils SHS front temperatures $T_{f_{1}}$ and $T_{f_{2}}$, it is possible to apply equation (2), solving it for parameter $\Delta y_{0}$ :

$$
\Delta y_{0}=\frac{\lambda_{1} \lambda_{2}\left(T_{f_{1}}-T_{f_{2}}\right)}{2\left[\left(T_{f_{1}}-T_{0}\right) \lambda_{1}-\left(T_{f_{2}}-T_{0}\right) \lambda_{2}\right]} .
$$

On the other hand, considering that structural and thermodynamic parameters can have an estimated value, it is important to determine the effectiveness of the influence of each of these parameters on foil reactivity. This enables not only forecasting its characteristics, depending on thermodynamic and structural parameters, but also assessing their «sensitivity» to the accuracy of these parameters determination.
Results and their discussion. To determine these values, the influence of thermodynamic and structural parameters was studied in the case of multilayer $\mathrm{Ni} / \mathrm{Al}$ foil of equiatomic composition. The same values of thermodynamic parameters of $\mathrm{Ni} / \mathrm{Al}$ system were taken as in [4]: $D_{0}=$ $=1.5 \cdot 10^{-5} \mathrm{~m}^{2} / \mathrm{s}, \quad Q=2.7 \cdot 10^{-19} \mathrm{~J}, a^{2}=$ $=7.42 \cdot 10^{-5} \mathrm{~m}^{2} / \mathrm{s}, \quad T_{0}=300 \mathrm{~K}, \quad \Delta g=$ $=7.36549 \cdot 10^{-20} \mathrm{~J}$.

In [4] it is shown that there exists a linear dependence of SHS reaction front temperature on foil effectiveness coefficient at different values of initial interlayer thickness. Let us consider the influence of thermodynamic parameters on SHS front temperature and its propagation velocity. With this purpose, SHS front temperature was calculated from equation (2), depending on thermodynamic stimulus at different structural parameters of the foil. It is seen (Figure 2) that SHS front temperature grows linearly at increase of thermodynamic stimulus, irrespective of foil structure. However, the level of SHS front temperature is essential influenced by foil structural characteristics. So, for instance, at $\Delta g=$ $=7.36549 \cdot 10^{-20} \mathrm{~J}$, SHS front temperature varies in the range from 1400 up to $2000 \mathrm{~K}$ at increase of multilayer period from 50 up to $300 \mathrm{~nm}$ and reduction of interlayer thickness from 8 to $4 \mathrm{~nm}$. In this connection, dependencies of front temperature on interlayer thickness $\Delta y_{0}$ and multilayer period $\lambda$ at constant value of thermodynamic stimulus were calculated. It is seen ( $\mathrm{Fi}^{-}$ gure 3) that for any multilayer period SHS front temperature drops at increase of interlayer thickness. Reduction of multilayer period also leads to lowering of SHS front temperature, and the stronger, the larger the intermediate layer thickness (Figure 4).

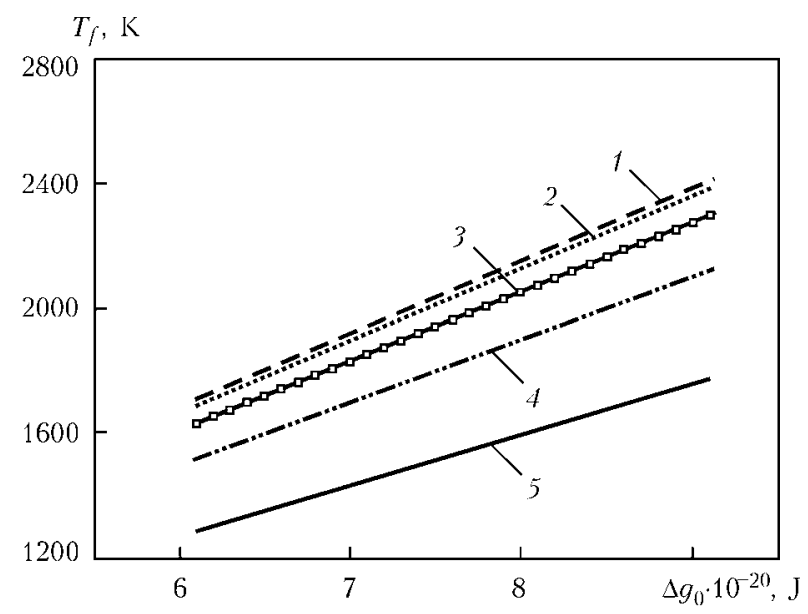

Figure 2. Dependence of SHS front temperature on thermodynamic stimulus: $1-\lambda=300 \mathrm{~nm}, \Delta y_{0}=4 \mathrm{~nm} ; 2-$ $\lambda=50 \mathrm{~nm}, \Delta y_{0}=1 \mathrm{~nm}, 3-\lambda=100 \mathrm{~nm}, \Delta y_{0}=4 \mathrm{~nm} ; 4-$ $\lambda=50 \mathrm{~nm}, \Delta y_{0}=4 \mathrm{~nm} ; 5-\lambda=50 \mathrm{~nm}, \Delta y_{0}=8 \mathrm{~nm}$ 


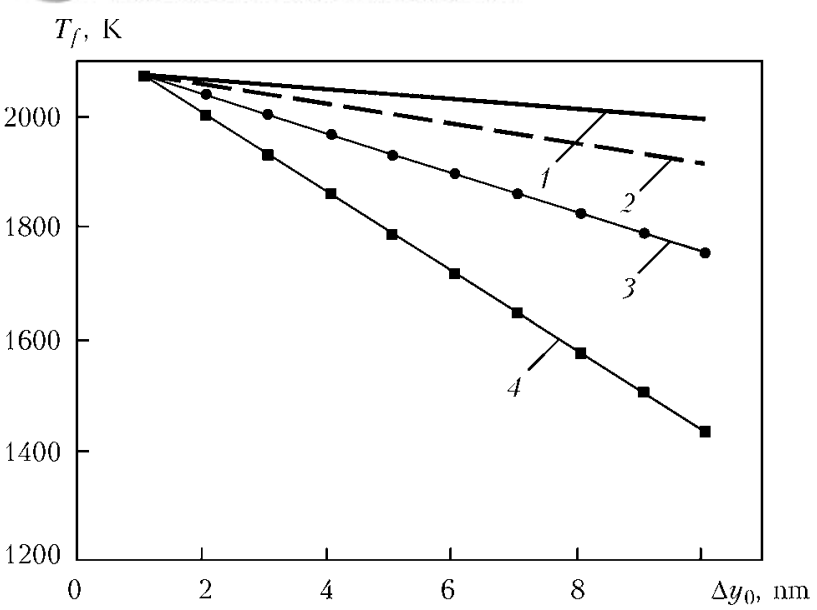

Figure 3. Dependence of SHS front temperature on interlayer thickness $\Delta y_{0}$ for $\mathrm{Ni} / \mathrm{Al}$ foil with different multilayer periods: $1-\lambda=400 ; 2-200 ; 3-100 ; 4-50 \mathrm{~nm}$

Thus, the conducted analysis showed that SHS front temperature depends both on thermodynamic stimulus, and on foil structural characteristics. In order to generalize such an interdependence, SHS front temperature dependencies on $\Delta g f$ value (furtheron referred to as thermodynamic coefficient of effectiveness of foil) were calculated. They are determined both by physical nature of elements forming the foil laminated structure, and by its structural characteristics. It is found that (Figure 5) dependence of SHS front temperature on thermodynamic coefficient of effectiveness is linear and invariant to structural characteristics.

As $\Delta g$ value is determined by the nature of elements forming the multilayer structure, increasing the thermodynamic coefficient of foil effectiveness requires increasing the volume fraction of elements, which did not enter into the reaction during foil preparation. According to (2), this can be achieved through reducing the interlayer thickness and increasing the multilayer period.

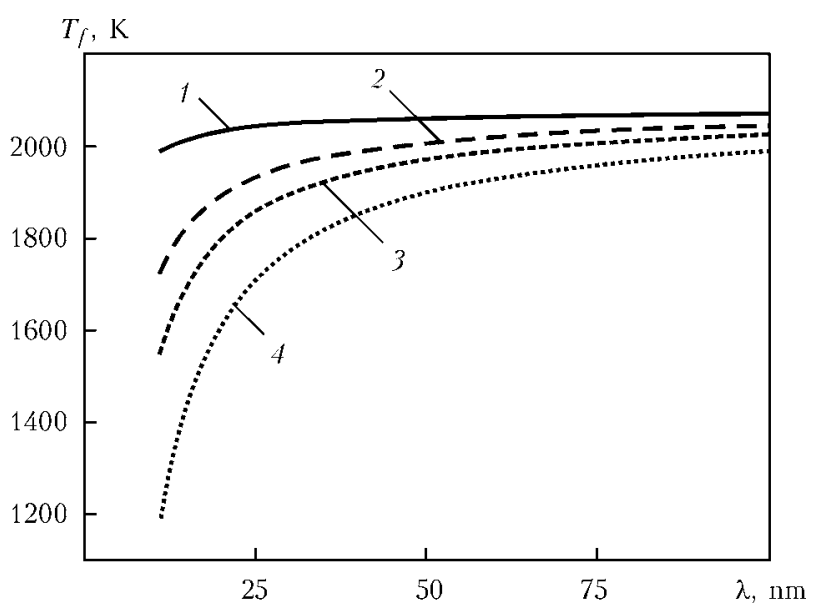

Figure 4. Dependence of SHS front temperature on multilayer period for $\mathrm{Ni} / \mathrm{Al}$ foil with different interlayer thicknesses: $1-\Delta y_{0}=1 ; 2-4 ; 3-6 ; 4-10 \mathrm{~nm}$

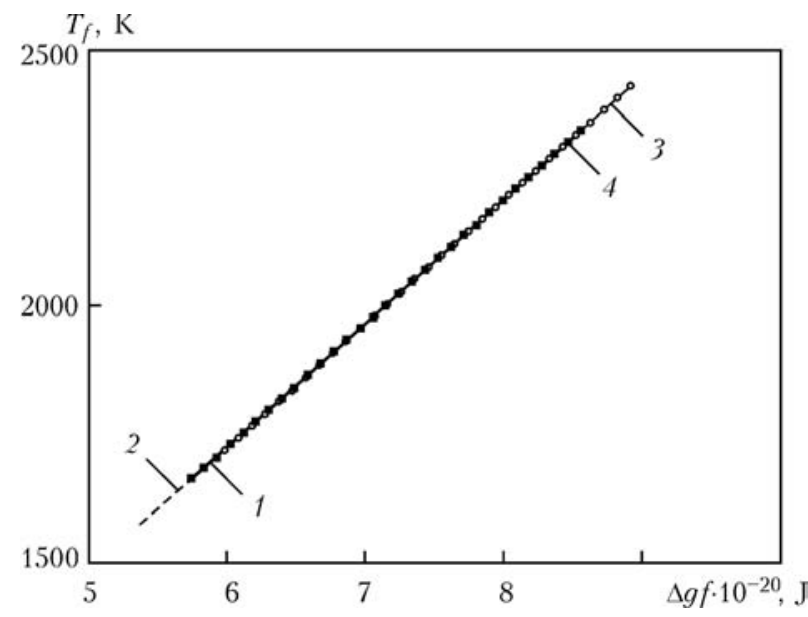

Figure 5. Dependence of combustion front temperature on thermodynamic coefficient of effectiveness of $\mathrm{Ni} / \mathrm{Al}$ foil: $1-\lambda=50 \mathrm{~nm}, \Delta y_{0}=1 \mathrm{~nm} ; 2-\lambda=50 \mathrm{~nm}, \Delta y_{0}=3 \mathrm{~nm}$; $3-\lambda=100 \mathrm{~nm}, \Delta y_{0}=1 \mathrm{~nm}, 4-\lambda=100 \mathrm{~nm}, \Delta y_{0}=3 \mathrm{~nm}$

On the other hand, at practical application of MF as a local heat source, not only SHS front temperature, but also its propagation velocity will determine foil effectiveness for local heating of the joint zone during reaction welding or brazing. At a low velocity of SHS front propagation, heat removal into the joint zone will decrease the foil capacity for local heating of this zone. Negative influence of this process can be reduced through increase of SHS front propagation velocity.

According to (1) thermodynamic stimulus is one of the parameters affecting the velocity of SHS front propagation. As is seen from Figure 6, the velocity of SHS front propagation is different from zero only in the case, when its magnitude is higher than a certain critical value, otherwise SHS reaction will not take place. It can be assumed that in systems, in which the thermodynamic stimulus is below a certain critical value,

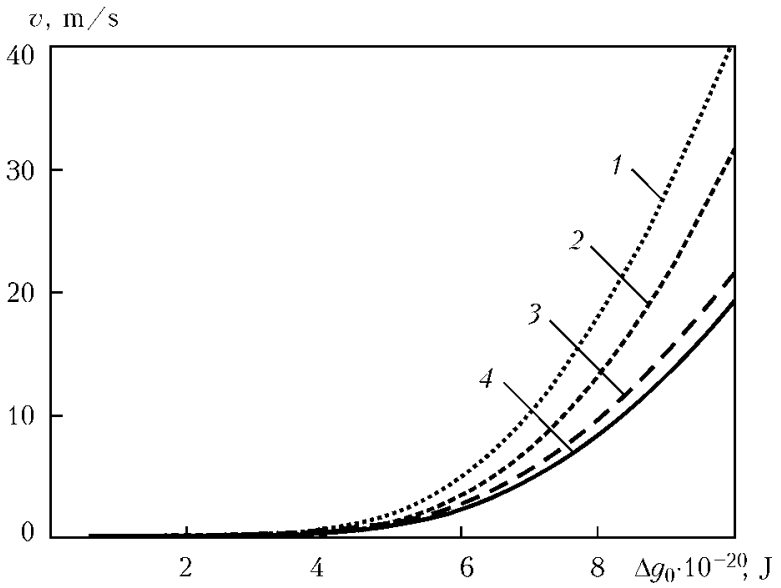

Figure 6. Dependence of reaction rate on thermodynamic stimulus for $\mathrm{Ni} / \mathrm{Al}$ foil: $1-\lambda=50 \mathrm{~nm}, \Delta y_{0}=1 \mathrm{~nm} ; 2-$ $\lambda=50 \mathrm{~nm}, \Delta y_{0}=3 \mathrm{~nm} ; 3-\lambda=100 \mathrm{~nm}, \Delta y_{0}=1 \mathrm{~nm} ; 4-$ $\lambda=100 \mathrm{~nm}, \Delta y_{0}=3 \mathrm{~nm}$ 


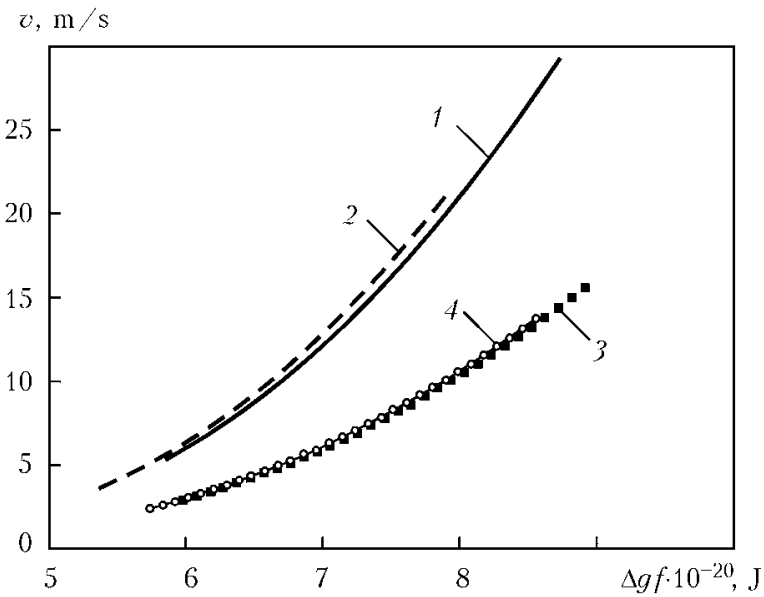

Figure 7. Dependence of reaction rate on thermodynamic coefficient of $\mathrm{Ni} / \mathrm{Al}$ foil effectiveness: $1-\lambda=50 \mathrm{~nm}$, $\Delta y_{0}=1 \mathrm{~nm} ; 2-\lambda=50 \mathrm{~nm}, \Delta y_{0}=3 \mathrm{~nm} ; 3-\lambda=100 \mathrm{~nm}$ $\Delta y_{0}=1 \mathrm{~nm} ; 4-\lambda=100 \mathrm{~nm}, \Delta y_{0}=3 \mathrm{~nm}$

it does not seem possible to realize the SHS reaction.

If thermodynamic stimulus is greater than this critical value, then at increase of thermodynamic coefficient of foil effectiveness the velocity of SHS front propagation will rise parabolically. The rate of this increase depends on foil structural characteristics: the smaller the multilayer period and the thinner the intermediate layer, the greater the intensity of SHS propagation velocity rise at increase of thermodynamic stimulus.

As seen from Figure 7, however, dependence of SHS front propagation velocity on thermodynamic coefficient of effectiveness is almost independent on interlayer thickness, and is determined just by multilayer period.

When the influence of foil microstructure parameters was studied (at unchanged value of thermodynamic parameters), it turned out (Figure 8) that for 3-4 nm thickness of interlayer inversion of the dependence of SHS front propagation velocity on multilayer period takes place - at smaller values of interlayer thickness front propagation velocity rises significantly at reduction of multilayer period, and at larger values it decreases.

Non-monotonic dependence of front propagation velocity is observed also at the change of multilayer period (Figure 9). It is seen that at reduction of multilayer period, SHS reaction front velocity rises, but, having reached a certain critical value, it decreases.

Thus, performed calculations show that to ensure the maximum velocity of SHS front propagation, the multilayer period should be determined allowing for interlayer thickness. Considering that this parameter has a specific value, which is determined by foil preparation condi- $v, \mathrm{~m} / \mathrm{s}$

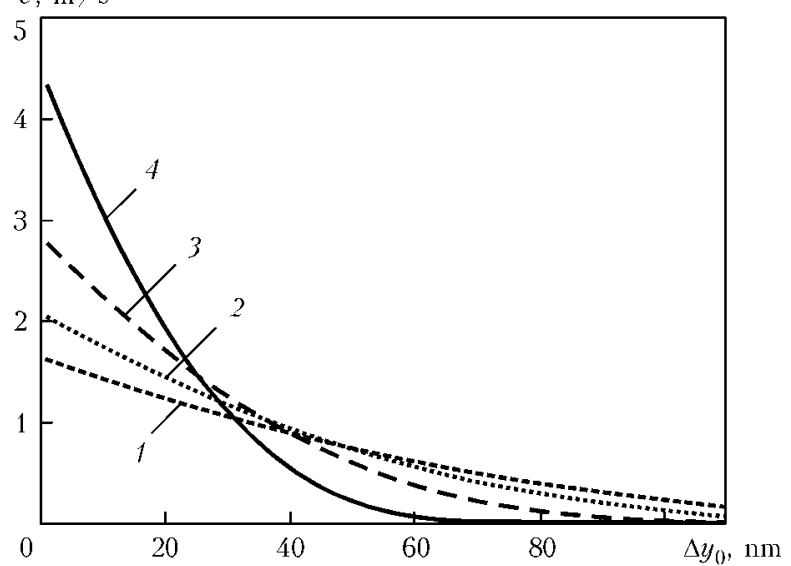

Figure 8. Dependence of reaction rate on interlayer thickness: $1-\lambda=480 ; 2-380 ; 3-280 ; 4-180 \mathrm{~nm}$

tions, SHS front propagation velocity can be varied by changing the multilayer period.

To determine the range of parameter values of MF microstructure, at which it will be capable of intensive heating of the joint zone, let us define heat generation intensity (HGI) $W$ in foil section of thickness $d(\mathrm{~cm})$, length $l=1 \mathrm{~cm}$ and width $m=1 \mathrm{~cm}$ at passage of SHS reaction front through it as

$$
W=\frac{Q}{S \tau},
$$

where $Q$ is the quantity of heat evolving in a foil region, which can be determined from $Q=C\left(T_{f}\right.$ $\left.-T_{0}\right) d \rho S$ relationship (here $C$ is the heat capacity of foil; $\rho$ is the foil specific weight; $S$ is the foil region area); $\tau$ is the time of front passage through foil region, which is determined as $\tau=$ $=l / v$. After substitution into (4) of values, included in the expressions, we will obtain

$$
W=\frac{C\left(T_{f}-T_{0}\right) d \rho v}{l}
$$

or allowing for (2)

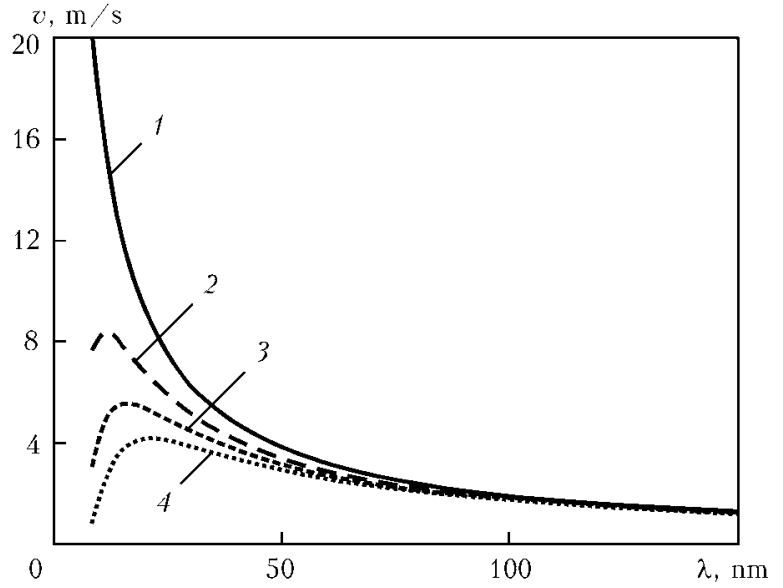

Figure 9. Dependence of combustion front propagation velocity on multilayer period: $1-\Delta y_{0}=1 ; 2-4 ; 3-6$; $4-8 \mathrm{~nm}$ 


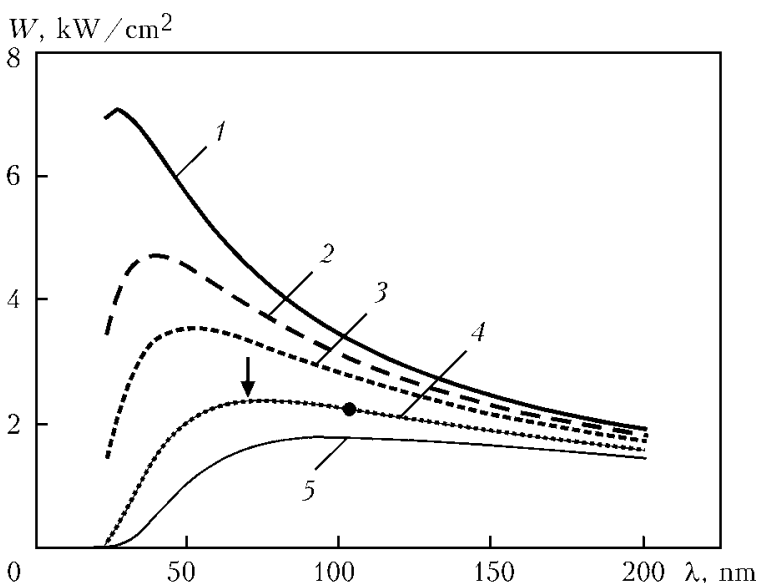

Figure 10. Dependence of HGI value during SHS reaction propagation in $26 \mu \mathrm{m} \mathrm{Ni} / \mathrm{AL}$ foil on multilayer period at different interlayer thicknesses: $1-\Delta y_{0}=2 ; 2-3 ; 3-$ $4 ; 4-6 ; 5-8 \mathrm{~nm}$

$$
W=\frac{C d \rho}{3 l k} \Delta g f v .
$$

It is seen from expression (6) that HGI value rises with increase of the coefficient of foil effectiveness $f$ and SHS front propagation velocity $v$. Considering, however, that the velocity of front propagation shows a non-monotonic dependence on structural parameters, it is anticipated that HGI dependence on these parameters will also be non-monotonic.

Indeed, as is seen from Figure 10, HGI value at reduction of multilayer period first rises, and then, having reached a certain value, starts decreasing, irrespective of interlayer thickness. Comparing $W$ dependencies on multilayer period $\lambda$, calculated at different values of interlayer thickness $\Delta y_{0}$, one can see that the maximum HGI value is the greater, the thinner the interlayer $\Delta y_{0}$.

Note the fact that the maximum position also depends on interlayer thickness: the thinner the interlayer, the smaller the multilayer period, at which maximum HGI value is reached.

It follows from the obtained results that if the thickness of interlayer formed under certain conditions of MF deposition, is known, then having plotted preliminary dependencies of $W$ on multilayer period $\lambda$ for different values of interlayer thickness, it is possible to find $\lambda_{\text {opt }}$, at which maximum HGI will be achieved.

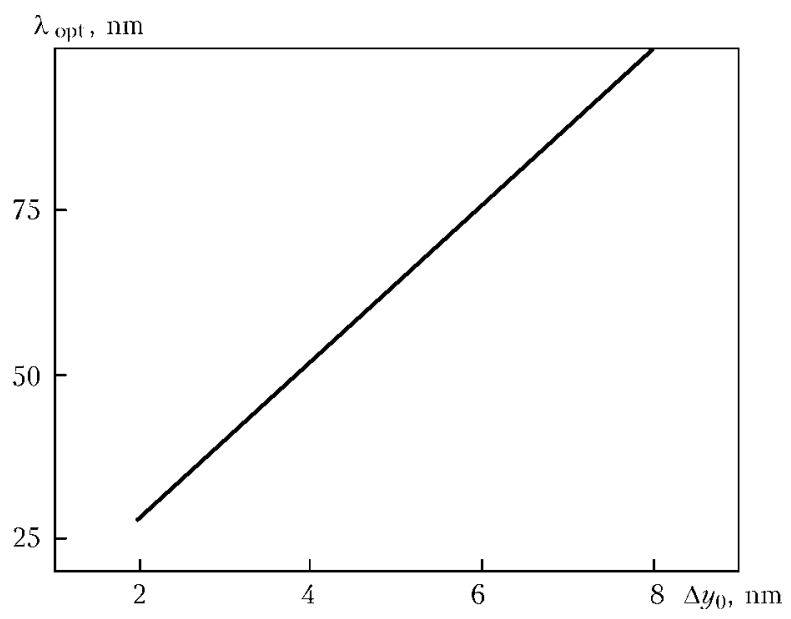

Figure 11. Dependence of multilayer optimal period $\lambda_{\mathrm{opt}}$ on interlayer thickness $\Delta y_{0}$ in $\mathrm{Ni} / \mathrm{Al}$ foil

Let us consider the possibility of $\lambda_{\text {opt }}$ determination based on experimental study of MF with different multilayer periods, produced under similar conditions. For this purpose, two foils with different multilayer periods were produced (under similar conditions) by the process of layerby-layer EB PVD [7] of nickel and aluminium. Foil characteristics and their deposition conditions are given in the Table, where $d$ is the foil thickness; $T_{\text {substr }}$ is the substrate temperature; $v_{\text {dep }}$ is the deposition rate.

Measurement of SHS front temperature $T_{f}$ and its propagation velocity $v_{f}$ was performed by the method presented in [8].

Interlayer thickness was calculated from equation (3). It was equal to a value of the order of $\Delta y_{0}=6.5 \mathrm{~nm}$. Proceeding from calculated dependence of $\lambda_{\text {opt }}$ on interlayer thickness $\Delta y_{0}$, given in Figure 11, one can see that under the given conditions of MF production, when $\Delta y_{0}=$ $=6.5 \mathrm{~nm}$, maximum HGI values can be equal to $2.3 \mathrm{~kW} / \mathrm{cm}^{2}$ under the condition that multilayer period $\lambda=\lambda_{\text {opt }}\left(\lambda_{\text {opt }} \approx 72-80 \mathrm{~nm}\right)$.

Condition for producing foil with maximum HGI can be also determined, if we use equation (5) to calculate HGI for foil with the smallest multilayer period (foil 1), and plot this value on the graph of $W$ dependence on multilayer period $\lambda$ (see Figure 10). One can see that the thus obtained experimental point can be correlated with one of $W$ dependencies on multilayer period $\lambda$, earlier calculated for MF with different interlayer thicknesses. Coincidence of the experimental point with one of the curves in Figure 10

Foil characteristics and deposition conditions

\begin{tabular}{|c|c|c|c|c|c|c|c||}
\hline \hline Foil numder & $d, \mu \mathrm{m}$ & $T_{\text {substr }}{ }^{\circ} \mathrm{C}$ & $v_{\text {dep }}, \mu \mathrm{m} / \mathrm{min}$ & $\lambda, \mathrm{nm}$ & $T_{f},{ }^{\circ} \mathrm{C}$ & $v_{f}, \mathrm{~m} / \mathrm{s}$ & $W, \mathrm{~kW} / \mathrm{cm}^{2}$ \\
\hline 1 & 25 & $220 \pm 10$ & 1.9 & 104 & 1160 & 1.23 & 2.21 \\
\hline 2 & 30 & $225 \pm 10$ & 1.9 & 520 & 1290 & 0.49 & 0.70 \\
\hline
\end{tabular}


allows determination of both the interlayer thickness, and $W$ maximum position, which corresponds to multilayer period of $72-80 \mathrm{~nm}$. Comparison of obtained $\lambda_{\text {opt }}$ values determined by various methods shows their satisfactory agreement.

One can see from theoretical analysis and experimental assessment of multilayer optimum period that factors promoting increase of interlayer thickness lead to increase of multilayer optimum period and lowering of HGI value.

\section{Conclusions}

1. Within the phenomenological model of stationary propagation of SHS reaction front in multilayer structure based on reaction elements of equiatomic composition, a procedure was proposed for determination of thickness of the interlayer, which forms in the foil during its production, by determination of SHS front temperature in two multilayer foils with different multilayer periods, produced under the same conditions.

2. It is shown that SHS front temperature becomes essentially dependent on interlayer thickness for multilayer structures with multilayer period less than $50 \mathrm{~nm}$.

3. It was confirmed that the temperature of SHS propagation front in the stationary mode is linearly dependent on thermodynamic coefficient of foil effectiveness.

4. It is shown that propagation of SHS front in the stationary mode is possible, if the thermodynamic coefficient of foil effectiveness exceeds a certain critical value, above which the velocity of SHS front propagation rises parabolically.
5. It is established that the maximum HGI level at SHS front propagation in multilayer foil is determined by interlayer thickness and multilayer period. At increase of interlayer thickness, multilayer period should be increased to provide maximum HGI.

1. (2003) Concept of SHS development as a field of scientific-technical progress. Ed. by A.G. Merzhanov. Chernogolovka: Territoriya.

2. Ustinov, A.I., Falchenko, Yu.V., Ishchenko, A.Ya. et al. (2008) Diffusion welding of TiAl alloys through nano-layered foil of $\mathrm{Ti} / \mathrm{Al}$ system. Intermetallic, $\mathbf{1 6}$, 1043-1045.

3. Rogachev, A.S., Grigoryan, A.E., Illarionova, E.V. et al. (2004) Gas-free burning of multilayer $\mathrm{Ti} / \mathrm{Al}$ bimetallic nanofilms. Fizika Goreniya $i$ Vzryva, $40(2), 45-51$

4. Zaporozhets, T.V., Gusak, A.M., Ustinov, A.I. (2010) Modeling of stationary mode of SHS reaction in nanolayered materials (phenomenological model). Pt 1: One-stage reaction. Sovrem. Elektrometallur giya, 1, 40-46.

5. Zaporozhets, T.V., Gusak, A.M., Ustinov, A.I. (2010) SHS reactions in nanosized multilayers Analytic model versus numeric model. Int. J. Self Propagating High Temperature Synthesis, 19(4), 227-236.

6. Zaporozhets, T.V. (2010) Modeling of stationary mode of SHS reaction propagation in nano-layered materials (phenomenological model). Pt 2: Two-stage reaction. Visnyk CherkGU, 185, 16-30.

7. Ustinov, A.I., Olikhovska, L.A., Melnichenko, T.V. et al. (2008) Effect of overall composition on thermally induced solid state transformations in thick EB PVD $\mathrm{Al} / \mathrm{Ni}$ multilayers. Surface and Coatings Techn., 202(16), 3832-3838.

8. Zaporozhets, T.V., Gusak, A.M., Korol, Ya.D. et al. (2013) Inverse problem for SHS in multilayer nanofoils: Prediction of process parameters for single-stage SHS reaction. Int. J. Self Propagating High Temperature Synthesis, 22(4), 217-225.

Received 28.04.2015 